\title{
Publisher Correction: Negative differential resistance as a critical indicator for the discharge capacity of lithium-oxygen batteries
}

\author{
Yoko Hase (1) ', Yasuhiro Komori², Takayoshi Kusumoto², Takashi Harada ${ }^{3}$, Juntaro Seki', Tohru Shiga (1) , \\ Kazuhide Kamiya ${ }^{2,3}$ \& Shuji Nakanishi ${ }^{2,3}$
}

Correction to: Nature Communications https://doi.org/10.1038/s41467-019-08536-z, published online: 05 February 2019

The original version of this Article contained an error in the title, which was previously incorrectly given as "Negative differential resistance as a critical indicator for the discharge capacity of lithium-oxygene batteries". The correct version states "lithium-oxygen" in place of "lithium-oxygene". This has been corrected in both the PDF and HTML versions of the Article.

Published online: 22 February 2019

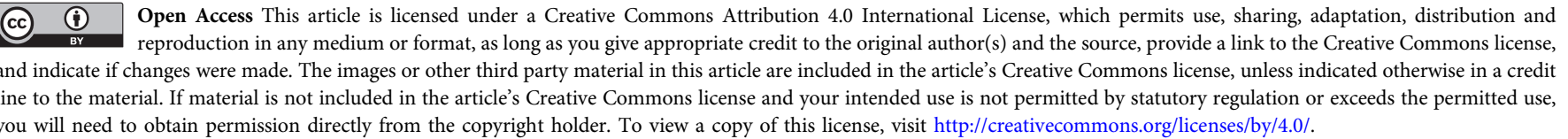
you will need to obtain permission directly from the copyright holder. To view a copy of this license, visit http://creativecommons.org/licenses/by/4.0/.

(c) The Author(s) 2019

\footnotetext{
${ }^{1}$ Toyota Central R\&D Labs., Inc., 41-1 Yokomichi, Nagakute, Aichi 480-1192, Japan. ${ }^{2}$ Research Center for Solar Energy Chemistry, Osaka University, 1-3 Machikaneyama, Toyonaka, Osaka 560-8531, Japan. ${ }^{3}$ Department of Chemistry, Graduate School of Engineering Science, Osaka University, 1-3

Machikaneyama, Toyonaka, Osaka 560-8531, Japan. Correspondence and requests for materials should be addressed to Y.H. (email: y-hase@mosk.tytlabs. co.jp) or to S.N. (email: nakanishi@chem.es.osaka-u.ac.jp)
} 